\title{
Scheme design of emergency communication network based on wireless ad hoc network
}

\author{
Feng Jing ${ }^{1, \text { a }}$, Hai-Yan Zhao ${ }^{1, b}$ \\ ${ }^{1}$ Xi'an Communication Institute, Xi'an 710106, China \\ a51375908@qq.com, b13086903@qq.com
}

\begin{abstract}
Key words: emergency communication network; Ad hoc network; wireless sensor network; WiMAX

Abstract: for the traditional emergency communication system can not meet the status of emergency communications needs, this article analyzed the emergency communication network and related technical means, and put forward two kinds of wireless ad hoc emergency communication network scheme, in order to provide technical reference for relevant emergency communication security department.
\end{abstract}

Emergency communication network is a temporary communication network to cope with the sudden large-scale natural disasters and public events. ECN can provide information services for disaster relief organizations, personnel and specific users by using strong communication capabilities to minimize disaster losses, maintain social stability, and support post disaster reconstruction. ECN is mainly used in emergencies such as floods, earthquakes, typhoons and other major natural disasters, as well as fire, public transport accidents and terrorist attacks and other ones.

At present, the domestic emergency communication technology is relatively backward, and the self-organized ability and survivability of the emergency communication system is undeveloped. With the rapid development of information and network technology, many new techniques gradually mature and show the characteristic in the field of emergency communication, especially the industry wide study of wireless ad hoc networks (wireless self organizing network, WSON) [1]. WSON is the organic combination of the mobile communication network and computer network, which has the characteristics of no infrastructure support, automatic network, multi hop relaying etc., network survivability and flexibility is stronger, especially suitable for battlefield, remote areas, emergency and temporary communication occasions.

\section{Emergency communication network main technical means}

\section{- Wireless ad hoc networks}

Wireless ad hoc network is a wireless (mobile) communication and computer network integration and development of the product and its characteristics is a network with no center, self-organizing, multi hop transmission, ad hoc networks and wireless sensor networks and wireless mesh network belong to the wireless ad hoc network category [2].

The mobile Ad hoc network is also a mobile ad hoc network, and all nodes in the network are composed of mobile hosts, when any two mobile hosts can communicate with each other in the communication coverage. At the same time, the mobile host also has the function of the router, and it can realize the remote data transmission through the routing function of the mobile host, which is particularly suitable for the formation of disaster emergency data transmission network ad hoc network with self-organized network, network node independence, dynamic network topology, high-speed mobile environment fast networking features [3]. It transmits the on-site information where people find difficult to reach to commanders on the scene.

Wireless sensor networks (wireless sensor networks, WSN) is a self-organizing wireless network made up of wireless sensors and the regional geography information can be sensed, acquired and processed of network coverage, and sent through the wireless network to the command center, for quick access to the scene of the accident information is of great significance. 
In recent years, due to features such as flexible networking, long data transmission distance, low cost, emergency communication field has seen rapid development. The emergency communication network has become one of the optional technical means because it can reach the area where human find difficult to reach, especially the network can be applied to the wiring difficulties,.

\section{- WiMAX broadband wireless network}

At the foundation of communication, a more effective way to provide broadband emergency communications is to use WiMAX wireless network technology, because it can meet the emergency communications network business and networking requirements. WiMAX is a broadband wireless communication technology based on IP, which can provide greater coverage ( $5 \sim 6 \mathrm{~km}$ ) and higher data rate (through the use of OFDMA, up and down up to a maximum of $\$ 70 \mathrm{~m}$ ), and is able to support high mobility $(100 \mathrm{~km} / \mathrm{h})$. In addition, WiMAX communication devices are easy to deploy and provide a certain QoS guarantees for different applications, such as emergency applications to give a higher priority and priority allocation of communication resources. Compared with 802.11 WLAN and 802.15 WPAN wireless communication technology, WiMAX has obvious technical advantages in the data rate, coverage and mobility support.

In order to support reliable communication in all kinds of emergency situations, the WiMAX network must be optimized to provide a wide range of non line of sight coverage and high speed mobility support. At the physical layer using OFDMA mechanism to improve system capacity, but the user mobility will bring Doppler frequency shift effect, also the carrier frequency shift (CFO) between the transmitter and the receiver will reduce the orthogonality between subcarriers and introduce inter carrier interference. Therefore, the clock synchronization accuracy should be improved to reduce the impact of mobility, and the compensation and feedback method is adopted to overcome the CFO introduced by MAI. In order to ensure reliable communication, a hybrid automatic repeat request (HARQ) technique with integrated ARQ and FEC is recommended. In addition, transmit diversity, beamforming and spatial multiplexing (MIMO) are used to improve the link reliability, network coverage and throughput.

\section{design of emergency communication network}

\section{- networking solutions based on Ad hoc}

Although the satellite communication system has the advantages of seamless coverage, but its networking capability is relatively weak. It is mainly designed for the voice and low speed data transmission of a single mobile user, so it is difficult to achieve a flexible network of large scale users. At the same time, the mobile Ad hoc network is a self-organizing network with a large number of nodes, which has a strong network capacity, but it is affected by the complexity of the routing and other factors, which affect the communication range of the network. Thus, we may be able to combine mobile ad hoc networks and satellite communication system effectively and construct a communication network based on satellite, ad hoc emergency [4], in which way can the satellite communication expand the communication range of the ad hoc, and use the flexibility of ad hoc networks to improve the emergency communication network of network capacity and mobility. Figure 1 shows the structure of the emergency field communication network which is combined with the satellite communication system and Ad hoc network.

As can be seen from Figure 1, through the Ad hoc network and on-site emergency communications vehicles deployed at the scene of the accident, the Ad hoc network can be effectively connected with the trunk network to achieve the interconnection between Ad hoc network and the external network. In this scenario, if the traditional land mobile communication network are not completely destroyed, the disaster information will be transmitted to the remote command center by traditional land mobile communication network; if the traditional land mobile communication system completely destroyed, disaster information can also be transmitted to the control center via a satellite link. when the ad hoc networks is used for disaster coverage, it can 
automatically adjust the network topology according to the compromised nodes, and the scene of the accident information will be transmitted to the superior command center, thereby greatly enhancing the ability of obtaining the scene of the accident information. At the same time, the effective utilization of the satellite transmission link can improve the information transmission of the disaster area and the command center. The on-site information can be transmitted at a long distance when the communication network is completely destroyed on the ground.

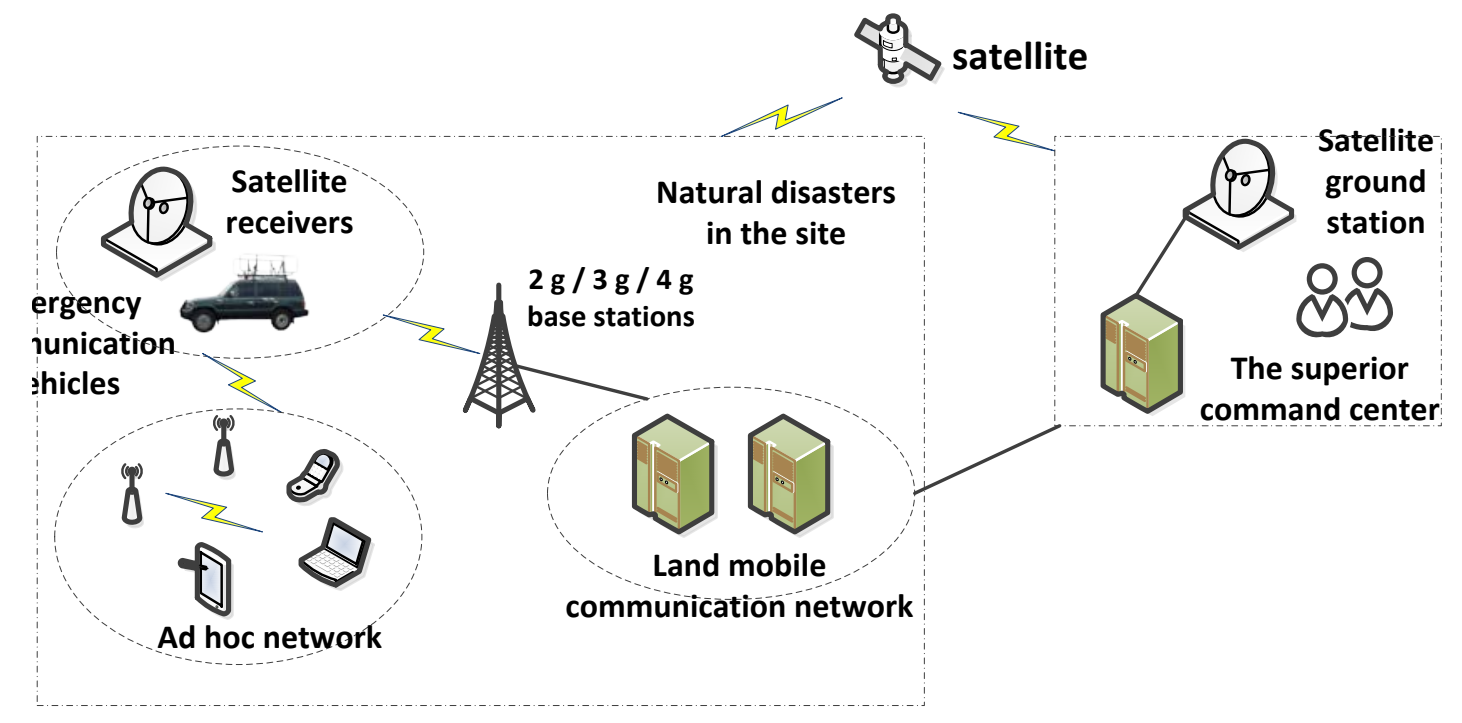

Figure1 networking solutions based on Ad hoc

\section{- networking solutions based on Wireless Sensor Networks}

When confronted with a severe natural disaster, for the harsh natural conditions, it is difficult for humans to arrive and rescue when the wireless sensor network can be adopted for real-time monitoring of the disaster area and monitoring information can be transmitted to the data points. At the same time, for satellite networks has high mobility and flexibility, large covering area and long communication transmission distance, wireless sensor network and satellite communication networks are combined, the wireless sensor network monitoring data using satellite communication link is transmitted in real time to superior command center. Figure 2 shows the diagram of the satellite sensor network architecture.

satellite

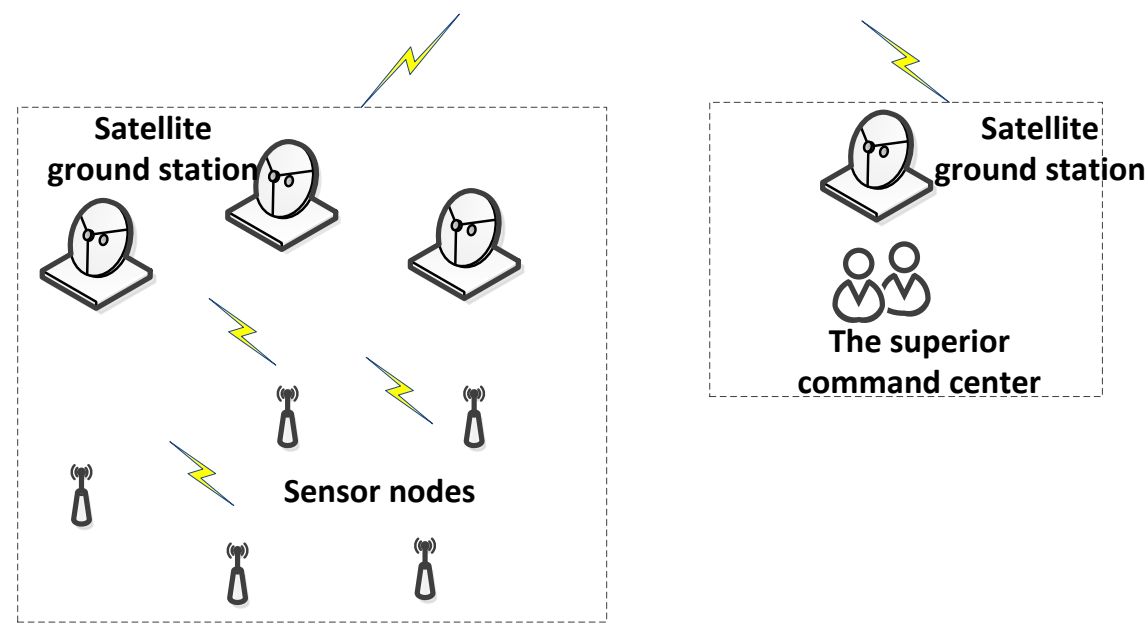

Figure2 emergency communication network scheme based on sensor 
In Figure 2, the main task of wireless sensor network is to detect the data of disaster area after natural disasters, and carry out a small range of data transmission in the area of wireless sensor network. Sensor nodes are usually deployed in the disaster areas to monitor the implementation of the accident detection, classification, tracking and reporting and other functions. Each sensor node include one or more sensors, taking into account the weight, cost and other requirements, the node should generally use the embedded processor, low power radar, etc.. At the same time, each node needs to have good power supply, taking into account the node power supply, sensor nodes should remain "silent" in the vast majority of cases and reach a fast delivery speed when monitoring data.

Emergency communication network based on wireless sensors and satellite communication effectively combines the advantages of existing terrestrial sensor networks and satellite communication system. It can not only solve the satellite communication earth station problems, but also be used effectively in sensor network flexible networking. It has features like low cost and other advantages to achieve the purpose of real-time monitoring of the disaster scene and exchange information between different regions.

\section{Conclusions}

The quick and effective construction of emergency communication network is very important for the major natural disasters and accidents emergency rescue work. As a supplementary means in the public communication network, the construction of emergency communication network should not be limited to conventional public communication network technology, and a variety of technical means, especially the effective use of wireless communication technology should be fully taken into consideration. Through the analysis on various existing emergency field communication network technology, it can be found that the emergency field communication network should give full play to the important role of satellite communication and mobile ad hoc networks. The information obtaining and long distance transmission should be taken into consideration in the construction of emergency communication network. The equipment mobility, network survivability, system reliability and service availability and other advantages of wireless ad hoc emergency communication network of communications should be made full advantages of.

\section{References}

[1] Xu S, Saadawi T. Does the IEEE 802.11 MAC Protocol Work Well in Multihop Wireless Ad Hoc Networks [J].IEEE Commun Mag, 2001,39(6):130-137.

[2] Tian Y, Xu K. TCP in Wireless Environments: Problems and Solutions[J].IEEE Commun Mag, 2005,43(3):27-32.

[3] Cai Z, Lu M, Wang X. Channel Access-Based Self-Organized Clustering in Ad Hoc Networks [J]. IEEE Trans Mobile Comp, 2003,2(2):102-113.

[4] Mauve M, Widme J. A Survey on Position-Based Routing in Mobile Ad Hoc Sensor Networks [J].IEEE Network,2001,15(3):30-39. 